\title{
Model Predictive Control of Uncertain Constrained Linear System Based on Mixed $\mathscr{H}_{2} / \mathscr{H}_{\infty}$ Control Approach
}

\author{
Patience E. Orukpe \\ Department of Electrical and Electronic Engineering, University of Benin, P.M.B 1154, Benin City, Edo State, Nigeria \\ Correspondence should be addressed to Patience E. Orukpe, patience.orukpe01@imperial.ac.uk
}

Received 30 June 2011; Revised 13 October 2011; Accepted 5 November 2011

Academic Editor: Marcin T. Cychowski

Copyright () 2012 Patience E. Orukpe. This is an open access article distributed under the Creative Commons Attribution License, which permits unrestricted use, distribution, and reproduction in any medium, provided the original work is properly cited.

Uncertain constrained discrete-time linear system is addressed using linear matrix inequality based optimization techniques. The constraints on the inputs and states are specified as quadratic constraints but are formulated to capture hyperplane constraints as well. The control action is of state feedback and satisfies the constraints. Uncertainty in the system is represented by unknown bounded disturbances and system perturbations in a linear fractional transform (LFT) representation. Mixed $\mathscr{H}_{2} / \mathscr{H}_{\infty}$ method is applied in a model predictive control strategy. The control law takes account of disturbances and uncertainty naturally. The validity of this approach is illustrated with two examples.

\section{Introduction}

Model predictive control (MPC) is a class of model-based control theories that use linear or nonlinear process models to forecast system behaviour. MPC is one of the control techniques that is able to cope with model uncertainties in an explicit way [1]. MPC has been used widely in practical applications to industrial process systems [2] and active vibration control of railway vehicles [3]. One of the methods used in MPC when uncertainties are present is to minimise the objective function for the worst possible case. This strategy is known as minimax and was originally proposed [4] in the context of robust receding control, [5] in the context of feedback and feedforward control and [6] in the context of $\mathscr{H}_{\infty}$ MPC. MPC has been applied to $\mathscr{H}_{\infty}$ problems in order to combine the practical advantage of MPC with the robustness of the $\mathscr{H}_{\infty}$ control, since robustness of MPC is still being investigated for it to be applied practically.

This work is motivated by the work in $[7,8]$ where uncertainty in the system was modeled by perturbations in a linear fractional representation. In [9], model predictive control based on a mixed $\mathscr{H}_{2} / \mathscr{H}_{\infty}$ control approach was considered. The designed controller has the form of state feedback and was constructed from the solution of a set of feasibility linear matrix inequalities. However, the issue of handling both uncertainty and disturbances simultaneously was not considered. In this paper, we extend the result of [9] to constrained uncertain linear discrete-time invariant systems using a mixed $\mathscr{H}_{2} / \mathscr{H}_{\infty}$ design approach and the uncertainty considered is norm-bounded additive. This is more suitable as both performance and robustness issues are handled within a unified framework.

The method presented in this paper develops an LMI design procedure for the state feedback gain matrix $F$, allowing input and state constraints to be included in a less conservative manner. A main contribution is the accomplishment of a prescribed disturbance attenuation in a systematic way by incorporating the well-known robustness guarantees through $\mathscr{H}_{\infty}$ constraints into the MPC scheme. In addition, norm-bounded additive uncertainty is also incorporated. A preliminary version of some of the work presented in this paper was presented in [10].

The structure of the work is as follows. After defining the notation, we describe the system and give a statement of the mixed $\mathscr{H}_{2} / \mathscr{H}_{\infty}$ problem in Section 2. In Section 3, we derive sufficient conditions, in the form of LMIs, for the existence of a state feedback control law that achieves the design specifications. In Section 4, we consider two examples that illustrate our algorithm. Finally, we conclude in Section 5. 
The notation we use is fairly standard. $\mathcal{R}$ denotes the set of real numbers, $\mathcal{R}^{n}$ denotes the space of $n$-dimensional (column) vectors whose entries are in $\mathcal{R}$ and $\mathcal{R}^{n \times m}$ denoting the space of all $n \times m$ matrices whose entries are in $\mathcal{R}$. For $A \in \mathcal{R}^{n \times m}$, we use the notation $A^{T}$ to denote transpose. For $x, y \in \mathcal{R}^{n}, x<y$ (and similarly $\leq,>$, and $\geq$ ) is interpreted element wise. The identity matrix is denoted as $I$ and the null matrix by 0 with the dimension inferred from the context.

\section{Problem Formulation}

We consider the following discrete-time linear time invariant system:

$$
\begin{aligned}
x_{k+1} & =A x_{k}+B_{w} w_{k}+B_{u} u_{k}+B_{p} p_{k}, \\
q_{k} & =C_{q} x_{k}+D_{q u} u_{k}+D_{q w} w_{k}, \\
p_{k} & =\Delta_{k} q_{k}, \\
z_{k} & =\left[\begin{array}{c}
C_{z} x_{k} \\
D_{z u} u_{k}
\end{array}\right],
\end{aligned}
$$

$x_{0}$ given,

where $x_{0}$ is the initial state, $x_{k} \in \mathcal{R}^{n}$ is the state, $w_{k} \in \mathcal{R}^{n_{w}}$ is the disturbance, $u_{k} \in \mathcal{R}^{n_{u}}$ is the control, $z_{k} \in \mathcal{R}^{n_{z}}$ is the controlled output, $A \in \mathcal{R}^{n \times n}, B_{w} \in \mathcal{R}^{n \times n_{w}}, B_{u} \in \mathcal{R}^{n \times n_{u}}, C_{z} \in$ $\mathcal{R}^{n_{z_{1}} \times n}$, and $D_{z u} \in \mathcal{R}^{n_{z_{2}} \times n_{u}}$, and where $n_{z}=n_{z_{1}}+n_{z_{2}}$. The signals $q_{k}$ and $p_{k}$ model uncertainties or perturbations appearing in the feedback loop.

The operator, $\Delta_{k}$, is block diagonal:

$$
\Delta_{k} \in \Delta_{k}=\left\{\Delta_{k}=\left[\begin{array}{ccc}
\Delta_{1 k} & & 0 \\
& \ddots & \\
0 & & \Delta_{t k}
\end{array}\right]:\left\|\Delta_{i k}\right\| \leq 1 \forall i\right\},
$$

and is norm bounded by one. Scalings can be included in $C_{q}$ and $B_{p}$, thus generalizing the bound. $\Delta_{k}$ can represent either a memoryless time-varying matrix with $\bar{\sigma}\left(\Delta_{i k}\right) \leq 1$, for $i=$ $1, \ldots, t, k \geq 0$, or the constraints:

$$
p_{i k}^{T} p_{i k} \leq q_{i k}^{T} q_{i k}, \quad i=1, \ldots, t,
$$

where $p_{k}=\left[p_{1 k}, \ldots, p_{t k}\right]^{T}, q_{k}=\left[q_{1 k}, \ldots, q_{t k}\right]^{T}$, and the partitioning is induced by $\Delta_{k}$. Each $\Delta_{k}$ is assumed to be either a full block or a repeated scalar block, and models a number of factors, such as dynamics or parameters, nonlinearities, that are unknown, unmodeled or neglected. In this work, we only consider full blocks for simplicity.

In terms of the state space matrices, this formulation can be viewed as replacing a fixed $\left(A, B_{u}, B_{w}\right)$ by $\left(A, B_{u}, B_{w}\right) \in$ $\left(\mathcal{A}, \mathcal{B}_{u}, \mathcal{B}_{w}\right)$, where

$$
\begin{aligned}
\left(\mathcal{A}, \mathcal{B}_{u}, \mathcal{B}_{w}\right)=\{ & {\left[A+B_{p} \Delta_{k} C_{q}, B_{u}+B_{p} \Delta_{k} D_{q u}, B_{w}\right.} \\
& \left.\left.+B_{p} \Delta_{k} D_{q w}\right] \mid \Delta_{k} \in \Delta_{k}\right\} .
\end{aligned}
$$

In robust model predictive control, we consider normbounded uncertainty and define stability in terms of quadratic stability [11] which requires the existence of a fixed quadratic Lyapunov function $\left(V(\zeta)=\zeta^{T} P \zeta, P>0\right)$ for all possible choices of the uncertainty parameters.

In the case of norm-bounded uncertainties:

$$
\begin{aligned}
& {\left[\begin{array}{lll}
A & B_{w} & B_{u}
\end{array}\right]} \\
& \quad \in\left\{\left[\begin{array}{lll}
A^{o} & B_{w}^{o} & B_{u}^{o}
\end{array}\right]+F_{A} \Delta_{H}\left[\begin{array}{lll}
E_{A} & E_{w} & E_{u}
\end{array}\right]: \Delta \in \Delta\right\},
\end{aligned}
$$

where $\left[\begin{array}{lll}A^{o} & B_{w}^{o} & B_{u}^{o}\end{array}\right]$ represents the nominal model, $\Delta_{H}=$ $\Delta(I-H \Delta)^{-1}$, with

$$
\begin{gathered}
\Delta \in \Delta:=\left\{\Delta=\operatorname{diag}\left(\delta_{1} I_{q_{1}}, \ldots, \delta_{l} I_{q_{l}}, \Delta_{l+1}, \ldots, \Delta_{l+f}\right):\|\Delta\|\right. \\
\left.\leq 1, \delta_{i} \in \mathcal{R}, \Delta_{i} \in \mathcal{R}^{q_{i} \times q_{i}}\right\}
\end{gathered}
$$

and where $F_{A}, E_{A}, E_{w}, E_{u}$, and $H$ are known and constant matrices with appropriate dimensions. This linear fractional representation of uncertainty, which is assumed to be well posed over $\Delta$ (i.e., $\operatorname{det}(I-H \Delta) \neq 0$ for all $\Delta \in \Delta$ ), has great generality and is used widely in robust control theory [12].

We use the following lemma, which is a slight modification of a result in [13] and which uses the fact that $\Delta \in \Delta$ to remove explicit dependence on $\Delta$ for the solution with norm bounded uncertainties.

Lemma 1. Let $\Delta$ be as described in (6) and define the subspaces

$$
\begin{gathered}
\Sigma=\left\{\operatorname{diag}\left(S_{1}, \ldots, S_{l}, \lambda_{1} I_{q l+1}, \ldots, \lambda_{s} I_{q l+f}\right)\right. \\
\left.: S_{i}=S_{i}^{T} \in \mathcal{R}^{q_{i} \times q_{i}}, \quad \lambda_{j} \in \mathcal{R}\right\}, \\
\Gamma=\left\{\operatorname{diag}\left(G_{1}, \ldots, G_{l}, 0_{q l+1}, \ldots, 0_{q l+f}\right): G_{i}=-G_{i}^{T} \in \mathcal{R}^{q_{i} \times q_{i}}\right\} .
\end{gathered}
$$

Let $T_{1}=T_{1}^{T}, T_{2}, T_{3}, T_{4}$ be matrices with appropriate dimensions. We have $\operatorname{det}\left(I-T_{4} \Delta\right) \neq 0$ and $T_{1}+T_{2} \Delta\left(I-T_{4} \Delta\right)^{-1} T_{3}+$ $T_{3}^{T}\left(I-\Delta^{T} T_{4}^{T}\right)^{-1} \Delta^{T} T_{2}^{T}<0$ for every $\Delta \in \Delta$ if there exist $S \in \mathbf{\Sigma}$ and $G \in \Gamma$ such that $S>0$ and

$$
\left[\begin{array}{cc}
T_{1}+T_{2} S T_{2}^{T} & T_{3}^{T}+T_{2} S T_{4}^{T}+T_{2} G \\
T_{3}+T_{4} S T_{2}^{T}+G^{T} T_{2}^{T} & T_{4} S T_{4}^{T}+T_{4} G+G^{T} T_{4}^{T}-S
\end{array}\right]<0 .
$$

If $\Delta$ is unstructured, then (8) becomes

$$
\left[\begin{array}{cc}
T_{1}+\lambda T_{2} T_{2}^{T} & T_{3}^{T}+\lambda T_{2} T_{4}^{T} \\
T_{3}+\lambda T_{4} T_{2}^{T} & \lambda\left(T_{4} T_{4}^{T}-I\right)
\end{array}\right]<0,
$$

for some scalar $\lambda>0$. In this case, condition (9) is both necessary and sufficient.

We also use the following Schur complement result [14].

Lemma 2. Let $X_{11}=X_{11}^{T}$ and $X_{22}=X_{22}^{T}$. Then

$$
\begin{aligned}
& {\left[\begin{array}{ll}
X_{11} & X_{12} \\
X_{12}^{T} & X_{22}
\end{array}\right] \geq 0 \Longleftrightarrow X_{22} \geq 0,} \\
& X_{22}-X_{12}^{T} X_{11}^{+} X_{12} \geq 0, \quad X_{12}\left(I-X_{11} X_{11}^{+}\right)=0,
\end{aligned}
$$

where $X_{11}^{+}$denotes the Moore-Penrose pseudo-inverse of $X_{11}$. 
We assume that the pair $\left(A, B_{u}\right)$ is stabilizable and that the disturbance is bounded as

$$
\|w\|_{2}:=\sqrt{\sum_{k=0}^{\infty} w_{k}^{T} w_{k}} \leq \bar{w}
$$

where $\bar{w} \geq 0$ is know.

The aim is to find a state feedback control law $\left\{u_{k}=F x_{k}\right\}$ in $\mathcal{L}_{2}$, where $F \in \mathcal{R}^{n_{u} \times n}$, such that the following constraints are satisfied for all $\Delta_{k} \in \Delta_{k}$.

(1) Closed-loop stability: the matrix $A+B_{u} F$ is stable.

(2) Disturbance rejection: for given $\gamma>0$, the transfer matrix from $w$ to $z$, denoted as $T_{z w}$, is quadratically stable and satisfies the $\mathscr{H}_{\infty}$ constraint

$$
\|z\|_{2}<\gamma\|w\|_{2}
$$

for $x_{0}=0$.

(3) Regulation: for given $\alpha>0$, the controlled output satisfies the $\mathscr{H}_{2}$ constraint:

$$
\|z\|_{2}:=\sqrt{\sum_{k=0}^{\infty} z_{k}^{T} z_{k}}<\alpha .
$$

(4) Input constraints: for given $H_{1}, \ldots, H_{m_{u}} \in \mathcal{R}^{n_{u} \times n_{u}}$, $H_{j}=H_{j}^{T} \geq 0, h_{1}, \ldots, h_{m_{u}} \in \mathcal{R}^{n_{u} \times 1}$, and $\bar{u}_{1}, \ldots, \bar{u}_{m_{u}} \in \mathcal{R}$, the inputs satisfy the quadratic constraints:

$$
u_{k}^{T} H_{j} u_{k}+2 h_{j}^{T} u_{k} \leq \bar{u}_{j}, \quad \forall k ; \text { for } j=1, \ldots, m_{u} \text {. }
$$

(5) State/output constraints: for given $G_{1}, \ldots, G_{m_{x}} \in$ $\mathcal{R}^{n \times n}, G_{j}=G_{j}^{T} \geq 0, g_{1}, \ldots, g_{m_{x}} \in \mathcal{R}^{n \times 1}$, and $\bar{x}_{1}, \ldots, \bar{x}_{m_{x}} \in$ $\mathcal{R}$ the states/outputs satisfy the quadratic constraints:

$$
x_{k+1}^{T} G_{j} x_{k+1}+2 g_{j}^{T} x_{k+1} \leq \bar{x}_{j}, \quad \forall k ; \text { for } j=1, \ldots, m_{x} .
$$

An $F \in \mathcal{R}^{n_{u} \times n}$ satisfying these requirements will be called an admissible state feedback gain.

\section{LMI Formulation of Sufficiency Conditions}

The next theorem, which is the main result of this paper, derives sufficient conditions, in the form of LMIs, for the existence of an admissible $F$.

Theorem 3. Let all variables, definitions, and assumptions be as above. Then there exists an admissible state feedback gain matrix $F$ if there exists solutions $Q=Q^{T} \in \mathcal{R}^{n \times n}, Y \in \mathcal{R}^{n_{u} \times n}$, $\delta_{j} \geq 0, \mu_{j} \geq 0, v_{j} \geq 0, \bar{\Lambda}=\operatorname{diag}\left(\bar{\lambda}_{1} I, \ldots, \bar{\lambda}_{t} I\right)>0$, and $\bar{\Psi}_{j}=\operatorname{diag}\left(\bar{\psi}_{1} I, \ldots, \bar{\psi}_{t} I\right)>0$ to the LMIs shown in (16)(19).

$$
\left[\begin{array}{ccccccc}
-Q & \star & \star & \star & \star & \star & \star \\
0 & -\alpha^{2} \gamma^{2} I & \star & \star & \star & \star & \star \\
0 & 0 & -\bar{\Lambda} & \star & \star & \star & \star \\
A Q+B_{u} Y & \alpha^{2} B_{w} & B_{p} \bar{\Lambda} & -Q & \star & \star & \star \\
C_{q} Q+D_{q u} Y & \alpha^{2} D_{q w} & 0 & 0 & -\bar{\Lambda} & \star & \star \\
C_{z} Q & 0 & 0 & 0 & 0 & -\alpha^{2} I & \star \\
D_{z u} Y & 0 & 0 & 0 & 0 & 0 & -\alpha^{2} I
\end{array}\right]<0,
$$

$$
\left[\begin{array}{ccc}
1 & \star & \star \\
\gamma^{2} \bar{w}^{2} & \alpha^{2} \gamma^{2} \bar{w}^{2} & \star \\
x_{0} & 0 & Q
\end{array}\right] \geq 0,
$$

$$
\left[\begin{array}{cccc}
\mathrm{Q} & \star & \star & \star \\
H_{j}^{1 / 2} Y & \mu_{j} I & \star & \star \\
-h_{j}^{T} Y & 0 & \mu_{j} \bar{u}_{j} & \star \\
0 & 0 & \mu_{j} & 1
\end{array}\right] \geq 0, \quad j=1, \ldots, m_{u}
$$

Here, $\star$ represents terms readily inferred from symmetry and the partitioning of $\bar{\Lambda}$ and $\bar{\Psi}_{j}$ is induced by the partitioning of $\Delta_{k}$. If such solutions exist, then $F=Y Q^{-1}$.
Remark 4. The variables in the LMI minimization of Theorem 3 are computed online at time $k$, the subscript $k$ is omitted for convenience. 
Proof. Using $u_{k}=F x_{k}$, the dynamics in (1) become

$$
x_{k+1}=\overbrace{\left(A+B_{u} F\right)}^{A_{c l}} x_{k}+B_{w} w_{k}+B_{p} p_{k}, \quad z_{k}=\overbrace{\left[\begin{array}{c}
C_{z} \\
D_{z u} F
\end{array}\right]}^{C_{c l}} x_{k} .
$$

Consider a quadratic function $V(x)=x^{T} P x, P>0$ of the state $x_{k}$. It follows from (20) that

$$
\begin{aligned}
V\left(x_{k+1}\right)-V\left(x_{k}\right) \\
=x_{k}^{T}\left[A_{c l}^{T} P A_{c l}-P\right] x_{k}+x_{k}^{T} A_{c l}^{T} P B_{w} w_{k}+x_{k}^{T} A_{c l}^{T} P B_{p} p_{k} \\
\quad+w_{k}^{T} B_{w}^{T} P A_{c l} x_{k}+w_{k}^{T} B_{w}^{T} P B_{w} w_{k}+w_{k}^{T} B_{w}^{T} P B_{p} p_{k} \\
\quad+p_{k}^{T} B_{p}^{T} P A_{c l} x_{k}+p_{k}^{T} B_{p}^{T} P B_{w} w_{k}+p_{k}^{T} B_{p}^{T} P B_{p} p_{k} \\
= \\
{\left[\begin{array}{lll}
x_{k}^{T} & w_{k}^{T} & p_{k}^{T}
\end{array}\right] K\left[\begin{array}{l}
x_{k} \\
w_{k} \\
p_{k}
\end{array}\right]-x_{k}^{T} C_{c l}^{T} C_{c l} x_{k}+\gamma^{2} w_{k}^{T} w_{k}, }
\end{aligned}
$$

where

$$
K=\left[\begin{array}{ccc}
A_{c l}^{T} P A_{c l}-P+C_{c l}^{T} C_{c l} & A_{c l}^{T} P B_{w} & A_{c l}^{T} P B_{p} \\
B_{w}^{T} P A_{c l} & B_{w}^{T} P B_{w}-\gamma^{2} I & B_{w}^{T} P B_{p} \\
B_{p}^{T} P A_{c l} & B_{p}^{T} P B_{w} & B_{p}^{T} P B_{p}
\end{array}\right] .
$$

Using $q_{k}=\left(C_{q}+D_{q u} F\right) x_{k}+D_{q w} w_{k}$,

$$
\begin{aligned}
q_{k}^{T} \Lambda q_{k}= & x_{k}^{T}\left(C_{q}+D_{q u} F\right)^{T} \Lambda\left(C_{q}+D_{q u} F\right) x_{k} \\
& +x_{k}^{T}\left(C_{q}+D_{q u} F\right)^{T} \Lambda D_{q w} w_{k} \\
& +w_{k}^{T} D_{q w}^{T} \Lambda\left(C_{q}+D_{q u} F\right) x_{k} \\
& +w_{k}^{T} D_{q w}^{T} \Lambda D_{q w} w_{k},
\end{aligned}
$$

where $\Lambda=\operatorname{diag}\left(\lambda_{1} I, \ldots \lambda_{t} I\right)$.

Substituting (23) into (21), it can be verified that we can write

$$
V\left(x_{k+1}\right)-V\left(x_{k}\right)=\left[\begin{array}{lll}
x_{k}^{T} & w_{k}^{T} & p_{k}^{T}
\end{array}\right] \bar{K}\left[\begin{array}{c}
x_{k} \\
w_{k} \\
p_{k}
\end{array}\right]+p_{k}^{T} \Lambda p_{k}
$$

$$
-q_{k}^{T} \Lambda q_{k}-x_{k}^{T} C_{c l}^{T} C_{c l} x_{k}+\gamma^{2} w_{k}^{T} w_{k}
$$

where $\bar{K}$ is defined in (25) and $C_{p w}:=C_{q}+D_{q u} F$.

$$
\bar{K}=\left[\begin{array}{ccc}
A_{c l}^{T} P A_{c l}-P+C_{c l}^{T} C_{c l}+C_{p w}^{T} \Lambda C_{p w} & A_{c l}^{T} P B_{w}+C_{p w}^{T} \Lambda D_{q w} & A_{c l}^{T} P B_{p} \\
B_{w}^{T} P A_{c l}+D_{q w}^{T} \Lambda C_{p w} & B_{w}^{T} P B_{w}-\gamma^{2} I+D_{q w}^{T} \Lambda D_{q w} & B_{w}^{T} P B_{p} \\
B_{p}^{T} P A_{c l} & B_{p}^{T} P B_{w} & B_{p}^{T} P B_{p}-\Lambda
\end{array}\right] .
$$

Assuming that $\lim _{k \rightarrow \infty} x_{k}=0$ we have

$$
\sum_{k=0}^{\infty}\left[x_{k+1}^{T} P x_{k+1}-x_{k}^{T} P x_{k}\right]=-x_{0}^{T} P x_{0} .
$$

We write the $\mathscr{H}_{2}$ cost function as

$$
\|z\|_{2}^{2}=\sum_{k=0}^{\infty}\left(x_{k}^{T} C_{c l}^{T} C_{c l} x_{k}-\gamma^{2} w_{k}^{T} w_{k}\right)+\gamma^{2} \sum_{k=0}^{\infty} w_{k}^{T} w_{k} .
$$

Adding (26) and (27) and carrying out a simple manipulation gives

$$
\begin{aligned}
\|z\|_{2}^{2}= & x_{0}^{T} P x_{0}+\gamma^{2}\|w\|_{2}^{2} \\
& +\sum_{k=0}^{\infty}\left[\begin{array}{lll}
x_{k}^{T} & w_{k}^{T} & p_{k}^{T}
\end{array}\right] \bar{K}\left[\begin{array}{l}
x_{k} \\
w_{k} \\
p_{k}
\end{array}\right]+\sum_{k=0}^{\infty}\left(p_{k}^{T} \Lambda p_{k}-q_{k}^{T} \Lambda q_{k}\right),
\end{aligned}
$$

where $\bar{K}$ is defined in (25).

Setting $x_{0}=0$, it follows from (3), (12), and (28) that $\|z\|_{2}<\gamma\|w\|_{2}$ if $\bar{K}<0$ and $\Lambda \geq 0$. In this work, we will take $\Lambda>0$ to simplify our solution [8]. Using (2) and Lemma 1 it can be shown that

$$
\bar{K}<0,
$$

is also sufficient for quadratic stability of $T_{z w}$. 
Next, we linearize the matrix inequality (29) by applying a Schur complement, to give

$$
\left[\begin{array}{ccccccc}
-P & \star & \star & \star & \star & \star & \star \\
0 & -\gamma^{2} I & \star & \star & \star & \star & \star \\
0 & 0 & -\Lambda & \star & \star & \star & \star \\
A_{c l} & B_{w} & B_{p} & -P^{-1} & \star & \star & \star \\
C_{p w} & D_{q w} & 0 & 0 & -\Lambda^{-1} & \star & \star \\
C_{z} & 0 & 0 & 0 & 0 & -I & \star \\
D_{z u} F & 0 & 0 & 0 & 0 & 0 & -I
\end{array}\right]<0
$$

Pre- and post-multiplying the equation above by $\operatorname{diag}\left(P^{-1}, I\right.$, $I, I, I, I, I)$ gives

$$
\left[\begin{array}{ccccccc}
-P^{-1} & \star & \star & \star & \star & \star & \star \\
0 & -\gamma^{2} I & \star & \star & \star & \star & \star \\
0 & 0 & -\Lambda & \star & \star & \star & \star \\
A_{c l} P^{-1} & B_{w} & B_{p} & -P^{-1} & \star & \star & \star \\
C_{p w} P^{-1} & D_{q w} & 0 & 0 & -\Lambda^{-1} & \star & \star \\
C_{z} P^{-1} & 0 & 0 & 0 & 0 & -I & \star \\
D_{z u} F P^{-1} & 0 & 0 & 0 & 0 & 0 & -I
\end{array}\right]<0,
$$

setting $Q=\alpha^{2} P^{-1}, F=Y P \alpha^{-2}=Y Q^{-1}, C_{p w}=C_{q}+D_{q u} F$ and multiplying through by $\alpha^{2}$, the equation above becomes

$$
\left[\begin{array}{ccccccc}
-Q & \star & \star & \star & \star & \star & \star \\
0 & -\alpha^{2} \gamma^{2} I & \star & \star & \star & \star & \star \\
0 & 0 & -\alpha^{2} \Lambda & \star & \star & \star & \star \\
A Q+B_{u} Y & \alpha^{2} B_{w} & \alpha^{2} B_{p} & -Q & \star & \star & \star \\
C_{q} Q+D_{q u} Y & \alpha^{2} D_{q w} & 0 & 0 & -\alpha^{2} \Lambda^{-1} & \star & \star \\
C_{z} Q & 0 & 0 & 0 & 0 & -\alpha^{2} I & \star \\
D_{z u} Y & 0 & 0 & 0 & 0 & 0 & -\alpha^{2} I
\end{array}\right]
$$
$<0$.

Pre- and post-multiplying the equation above by $\operatorname{diag}(I, I$, $\left.\Lambda^{-1}, I, I, I, I\right)$ gives

$$
\left[\begin{array}{ccccccc}
-Q & \star & \star & \star & \star & \star & \star \\
0 & -\alpha^{2} \gamma^{2} I & \star & \star & \star & \star & \star \\
0 & 0 & -\alpha^{2} \Lambda^{-1} & \star & \star & \star & \star \\
A Q+B_{u} Y & \alpha^{2} B_{w} & \alpha^{2} B_{p} \Lambda^{-1} & -Q & \star & \star & \star \\
C_{q} Q+D_{q u} Y & \alpha^{2} D_{q w} & 0 & 0 & -\alpha^{2} \Lambda^{-1} & \star & \star \\
C_{z} Q & 0 & 0 & 0 & 0 & -\alpha^{2} I & \star \\
D_{z u} Y & 0 & 0 & 0 & 0 & 0 & -\alpha^{2} I
\end{array}\right]
$$$$
<0 \text {. }
$$

The equation above is a bilinear matrix inequality, thus by defining $\alpha^{2} \Lambda^{-1}$ as a variable $\bar{\Lambda}$, we get the LMI in (16).

Now, it follows from (3), (11), (28), and (29) that,

$$
\|z\|_{2}^{2} \leq x_{0}^{T} P x_{0}+\gamma^{2}\|w\|_{2}^{2} \leq x_{0}^{T} P x_{0}+\gamma^{2} \bar{w}^{2} .
$$

Thus the $\mathscr{H}_{2}$ constraint in (13) is satisfied if

$$
x_{0}^{T} P x_{0}+\gamma^{2} \bar{w}^{2}<\alpha^{2}
$$

Dividing by $\alpha^{2}$, rearranging and using a Schur complement give (17) as an LMI sufficient condition for (13).

To turn (14) and (15) into LMIs, we first show that $x_{k}^{T} P x_{k} \leq \alpha^{2} \forall k>0$. Since $\bar{K}<0$, it follows from (3) and (24) that

$$
x_{k+1}^{T} P x_{k+1}-x_{k}^{T} P x_{k} \leq \gamma^{2} w_{k}^{T} w_{k}
$$

Applying this inequality recursively, we get

$$
\begin{aligned}
x_{k}^{T} P x_{k} & \leq x_{0}^{T} P x_{0}+\gamma^{2} \sum_{j=0}^{k-1} w_{j}^{T} w_{j} \\
& \leq x_{0}^{T} P x_{0}+\gamma^{2} \bar{w}^{2}<\alpha^{2} .
\end{aligned}
$$

It follows that

$$
\left\|P^{1 / 2} x_{k}\right\|^{2}<\alpha^{2}
$$

or equivalently,

$$
x_{k}^{T} Q^{-1} x_{k}<1, \quad \forall k>0 .
$$

Next, we transform the constraints in (14) to a set of LMIs. Setting $F=Y Q^{-1}=Y P \alpha^{-2}$ and $u_{k}=F x_{k}$,

$$
\begin{aligned}
e_{j}\left(u_{k}\right) & :=u_{k}^{T} H_{j} u_{k}+2 h_{j}^{T} u_{k}-\bar{u}_{j} \\
& =x_{k}^{T} Q^{-1} Y^{T} H_{j} Y Q^{-1} x_{k}+2 h_{j}^{T} Y Q^{-1} x_{k}-\bar{u}_{j} .
\end{aligned}
$$

Now for any $\mu_{j} \in \mathcal{R}$, we can write

$$
\begin{aligned}
& e_{j}\left(u_{k}\right)=-\mu_{j}\left(1-x_{k}^{T} Q^{-1} x_{k}\right)-\left[\begin{array}{c}
x_{k} \\
1
\end{array}\right]^{T} \\
& \times\left(\left[\begin{array}{ccc}
\mu_{j} Q^{-1} & -Q^{-1} Y^{T} H_{j} Y Q^{-1} & -Q^{-1} Y^{T} h_{j} \\
& -h_{j}^{T} Y Q^{-1} & -\mu_{j}+\bar{u}_{j}
\end{array}\right]\right) \\
& \times\left[\begin{array}{c}
x_{k} \\
1
\end{array}\right] \text {. }
\end{aligned}
$$



and

Therefore a sufficient condition for $\mathrm{e}_{j}\left(u_{k}\right) \leq 0$ is $\mu_{j} \geq 0$

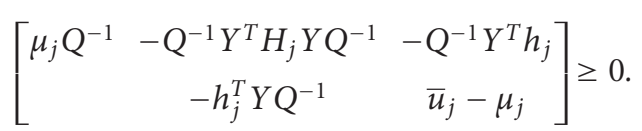

Pre- and post-multiplying by $\operatorname{diag}(Q, I)$ gives a bilinear matrix inequality and applying a Schur complement, we get

$$
\left[\begin{array}{ccc}
\mu_{j} Q & Y^{T} H_{j}^{1 / 2} & -Y^{T} h_{j} \\
H_{j}^{1 / 2} Y & I & 0 \\
-h_{j}^{T} Y & 0 & \bar{u}_{j}-\mu_{j}
\end{array}\right] \geq 0 .
$$

Pre- and post-multiplying the above bilinear matrix inequality by $\operatorname{diag}\left(\mu_{j}^{-1 / 2}, \mu_{j}^{1 / 2}, \mu_{j}^{1 / 2}\right)$ and applying a Schur complement, this is equivalent to the LMI in (18).
Finally, to obtain an LMI formulation of the state constraints (15), the following analogous steps are carried out:

$$
\begin{aligned}
f_{j}\left(x_{k+1}\right):= & {\left[\begin{array}{c}
x_{k} \\
w_{k} \\
p_{k}
\end{array}\right]^{T}\left[\begin{array}{c}
A_{c l}^{T} \\
B_{w}^{T} \\
B_{p}^{T}
\end{array}\right] G_{j}\left[\begin{array}{lll}
A_{c l} & B_{w} & B_{p}
\end{array}\right]\left[\begin{array}{c}
x_{k} \\
w_{k} \\
p_{k}
\end{array}\right] } \\
& +2 g_{j}^{T}\left[\begin{array}{lll}
A_{c l} & B_{w} & B_{p}
\end{array}\right]\left[\begin{array}{c}
x_{k} \\
w_{k} \\
p_{k}
\end{array}\right]-\bar{x}_{j} .
\end{aligned}
$$

$$
\begin{aligned}
f_{j}\left(x_{k+1}\right)= & -v_{j}\left(1-x_{k}^{T} Q^{-1} x_{k}\right)-\rho_{j}\left(\bar{w}^{2}-w_{k}^{T} w_{k}\right) \\
& -\left[\begin{array}{c}
x_{k} \\
w_{k} \\
1
\end{array}\right]^{T}\left(\left[\begin{array}{ccc}
v_{j} Q^{-1}-\left(A_{c l}+B_{p} \Delta_{k} C_{p w}\right)^{T} G_{j}\left(A_{c l}+B_{p} \Delta_{k} C_{p w}\right) & \star & \star \\
-\left(B_{w}+B_{p} \Delta_{k} D_{q w}\right)^{T} G_{j}\left(A_{c l}+B_{p} \Delta_{k} C_{p w}\right) & \rho_{j} I \\
-g_{j}^{T}\left(A_{c l}+B_{p} \Delta_{k} C_{p w}\right) & -g_{j}^{T}\left(B_{w}+B_{p} \Delta_{k} D_{q w}\right) & -v_{j}-\rho_{j} \bar{w}^{2}+\bar{x}_{j}
\end{array}\right]\right)\left[\begin{array}{c}
x_{k} \\
w_{k} \\
1
\end{array}\right] .
\end{aligned}
$$

Therefore a sufficient condition for $f_{j}\left(x_{k+1}\right) \geq 0$ is $v_{j} \geq 0$, $\rho_{j} \geq 0$ and

$$
\begin{aligned}
& {\left[\begin{array}{ccc}
v_{j} Q^{-1} & \star & \star \\
0 & \rho_{j} I & \star \\
-g_{j}^{T}\left(A_{c l}+B_{p} \Delta_{k} C_{p w}\right) & -g_{j}^{T}\left(B_{w}+B_{p} \Delta_{k} D_{q w}\right) & \bar{x}_{j}-v_{j}-\rho_{j} \bar{w}^{2}
\end{array}\right]} \\
& -\left[\begin{array}{c}
\left(A_{c l}+B_{p} \Delta_{k} C_{p w}\right)^{T} \\
\left(B_{w}+B_{p} \Delta_{k} D_{q w}\right)^{T} \\
0
\end{array}\right] G_{j}\left[\begin{array}{lll}
\left(A_{c l}+B_{p} \Delta_{k} C_{p w}\right) & \left(B_{w}+B_{p} \Delta_{k} D_{q w}\right) & 0
\end{array}\right] \geq 0 .
\end{aligned}
$$

Applying Schur complement to the above equation, we get

$$
\left[\begin{array}{cccc}
v_{j} Q^{-1} & \star & \star & \star \\
0 & \rho_{j} I & \star & \star \\
-g_{j}^{T}\left(A_{c l}+B_{p} \Delta_{k} C_{p w}\right) & -g_{j}^{T}\left(B_{w}+B_{p} \Delta_{k} D_{q w}\right) & \bar{x}_{j}-v_{j}-\rho_{j} \bar{w}^{2} & \star \\
G_{j}^{1 / 2}\left(A_{c l}+B_{p} \Delta_{k} C_{p w}\right) & G_{j}^{1 / 2}\left(B_{w}+B_{p} \Delta_{k} D_{q w}\right) & 0 & I
\end{array}\right] \geq 0 .
$$


When $\Delta$ is structured we proceed as follows. For normbounded uncertainty, we first separate the terms involving modeling uncertainties from the other terms as

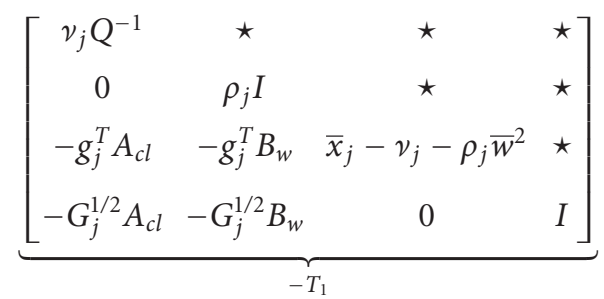

$+\underbrace{\left[\begin{array}{c}0 \\ 0 \\ -g_{j}^{T} B_{p} \\ G_{j}^{1 / 2} B_{p}\end{array}\right]}_{-T_{2}} \Delta_{k} \underbrace{\left[\begin{array}{llll}C_{p w} & D_{q w} & 0 & 0\end{array}\right]}_{T_{3}}$

$$
+\underbrace{\left[\begin{array}{c}
C_{p w}^{T} \\
D_{q w}^{T} \\
0 \\
0
\end{array}\right]}_{T_{3}^{T}} \Delta_{k}^{T} \underbrace{\left[\begin{array}{llll}
0 & 0 & -B_{p}^{T} g_{j} & B_{p}^{T} G^{1 / 2}
\end{array}\right]}_{-T_{2}^{T}} \geq 0 .
$$

Equation (48) is equivalent to $-T_{1}-T_{2} \Delta T_{3}-T_{3}^{T} \Delta^{T} T_{2}^{T}>0$, where $T_{4}=0$. By using (8) from Lemma 1, we have

$$
\left[\begin{array}{cc}
-T_{1}-T_{3}^{T} S T_{3} & -T_{2} \\
-T_{2}^{T} & S
\end{array}\right]>0 .
$$

Applying Schur complement to (49), we get

$$
\left[\begin{array}{ccc}
-T_{1} & T_{3}^{T} & -T_{2} \\
T_{3} & S^{-1} & 0 \\
-T_{2}^{T} & 0 & S
\end{array}\right]>0 .
$$

Substituting the variables from (48) into (50) and swapping the third and sixth diagonal elements, we get

Pre- and post-multiplying by $\operatorname{diag}(Q, I, I, I, I, I)$ gives

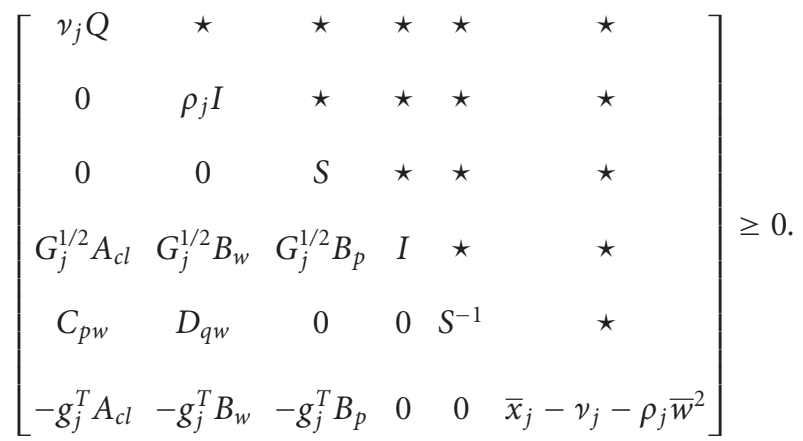

The above equation is bilinear and thus we pre- and post-multiply it by $\operatorname{diag}\left(v_{j}^{-1 / 2}, v_{j}^{1 / 2}, v_{j}^{1 / 2}, v_{j}^{1 / 2}, v_{j}^{1 / 2}, v_{j}^{1 / 2}\right)$ to obtain

$$
\left[\begin{array}{cccccc}
Q & \star & \star & \star & \star & \star \\
0 & v_{j} \rho_{j} I & \star & \star & \star & \star \\
0 & 0 & v_{j} S & \star & \star & \star \\
G_{j}^{1 / 2} A_{c l} & v_{j} G_{j}^{1 / 2} B_{w} & v_{j} G_{j}^{1 / 2} B_{p} & v_{j} I & \star & \star \\
C_{p w} & v_{j} D_{q w} & 0 & 0 & v_{j} S^{-1} & \star \\
-g_{j}^{T} A_{c l} & -v_{j} g_{j}^{T} B_{w} & -v_{j} g_{j}^{T} B_{p} & 0 & 0 & v_{j} \bar{x}_{j}-v_{j}^{2}-v_{j} \rho_{j} \bar{w}^{2}
\end{array}\right] \geq 0 .
$$

From the above equation, we can see that the variable $S$ and its inverse appear in the matrix inequality, thus to make it uniform, we pre- and post-multiply by $\operatorname{diag}\left(I, I, S^{-1}, I, I, I\right)$ to get 


$$
\left[\begin{array}{cccccc}
Q & \star & \star & \star & \star & \star \\
0 & v_{j} \rho_{j} I & \star & \star & \star & \star \\
0 & 0 & v_{j} S^{-1} & \star & \star & \star \\
G_{j}^{1 / 2} A_{c l} & v_{j} G_{j}^{1 / 2} B_{w} & v_{j} G_{j}^{1 / 2} B_{p} S^{-1} & v_{j} I & \star & \star \\
C_{p w} & v_{j} D_{q w} & 0 & 0 & v_{j} S^{-1} & \star \\
-g_{j}^{T} A_{c l} & -v_{j} g_{j}^{T} B_{w} & -v_{j} g_{j}^{T} B_{p} S^{-1} & 0 & 0 & v_{j} \bar{x}_{j}-v_{j}^{2}-v_{j} \rho_{j} \bar{w}^{2}
\end{array}\right] \geq 0 .
$$

Thus the above equation is a nonlinear matrix inequality in $v_{j}^{2}$ and bilinear in $v_{j} \rho_{j}$ and $v_{j} S^{-1}$, hence we define new variables $\bar{\Psi}_{j}=v_{j} S^{-1}$ and $\delta_{j}=v_{j} \rho_{j}$ and finally applying a Schur complement, we obtain the LMI of (19).

Remark 5. The input and state constraints used in this paper are more general than those used in [9] in that we allow linear terms and so this makes it possible to include asymmetric or hyperplane constraints.

Remark 6. When there is no uncertainty, the problem reduces to disturbance rejection technique considered in [9].

Remark 7. When there is no disturbance, the results reduce to those of [7].

Remark 8. The method used in this paper guarantees recursive feasibility (see [15, Chapter 4]). Also see [16] for a different approach.

\section{Numerical Examples}

In this section, we present two examples that illustrate the implementation of the proposed scheme. In the first example we consider a solenoid system, and in the second example we consider the coupled spring-mass system. The solution to the linear objective minimization was computed using LMI Control Toolbox in the MATLAB ${ }^{\circledR}$ environment and $\alpha^{2}$ was set as a variable.

4.1. Example 1. We consider a modified version of the solenoid system adapted from [17]. The system (see Figure 1) consists of a central object wrapped with coil and is attached to a rigid surface via a spring and damper, which forms a passive vibration isolator. The solenoid is one of the common actuator components. The basic principle of operation involves a moving ferrous core (a piston) that moves inside a wire coil. Normally, the piston is held outside the core by a spring and damper. When a voltage is applied to the coil and current flows, the coil builds up a magnetic field that attracts the piston and pulls it into the center of the coil. The piston can be used to supply a linear force. Application of this includes pneumatic valves and car door openers.
The system is modeled by

$$
\begin{aligned}
{\left[\begin{array}{c}
x_{k+1}^{1} \\
x_{k+1}^{2}
\end{array}\right]=} & {\left[\begin{array}{cc}
0.6148 & 0.0315 \\
-0.3155 & -0.0162
\end{array}\right]\left[\begin{array}{l}
x_{k}^{1} \\
x_{k}^{2}
\end{array}\right]+\left[\begin{array}{l}
0.0385 \\
0.0315
\end{array}\right] u_{k} } \\
& +\left[\begin{array}{l}
0.00385 \\
0.00315
\end{array}\right] w_{k}+\left[\begin{array}{c}
0 \\
10
\end{array}\right] p_{k}, \\
q_{k}= & C_{q} x_{k}+D_{q u} u_{k}+D_{q w} w_{k} \\
p_{k}= & \Delta q_{k}, \\
z_{k}= & {\left[\begin{array}{c}
C_{z} x_{k} \\
D_{z u} u_{k}
\end{array}\right] }
\end{aligned}
$$

where

$$
C_{q}=\left[\begin{array}{ll}
1 & 0
\end{array}\right], \quad D_{q u}=1, \quad D_{q w}=0
$$

where $x^{1}$ and $x^{2}$ are the position and the velocity of the plate. The cost function is specified using $C_{z}=\operatorname{diag}(1,1)$ and $D_{z u}=10$. The magnetic force $u$ is the control variable, and $w$ is the external disturbance to the system, which is bounded in the range $[-1,1]$. The initial state is given as $x_{0}=\left[\begin{array}{ll}1 & 0\end{array}\right]^{T}$.

We choose $\gamma^{2}=0.01$ and $\gamma^{2}=1$. Figures 2 and 3 compare the closed-loop response for the high and low disturbance rejection levels, respectively, for randomly generated $\Delta$ 's. The optimization is feasible, the response is stable, and the performance is good. A control constraint of $\left|u_{k}\right| \leq 0.5$ is imposed, which is satisfied. The computation time for 100 samples was about $10 \mathrm{~s}$, making $0.1 \mathrm{~s}$ per sample.

4.2. Example 2. We revisit a modified version of Example 2 reported in [7]. The system consists of a two-mass-spring model whose discrete-time equivalent is obtained using Euler first-order approximation with a sampling time of $0.1 \mathrm{~s}$. 


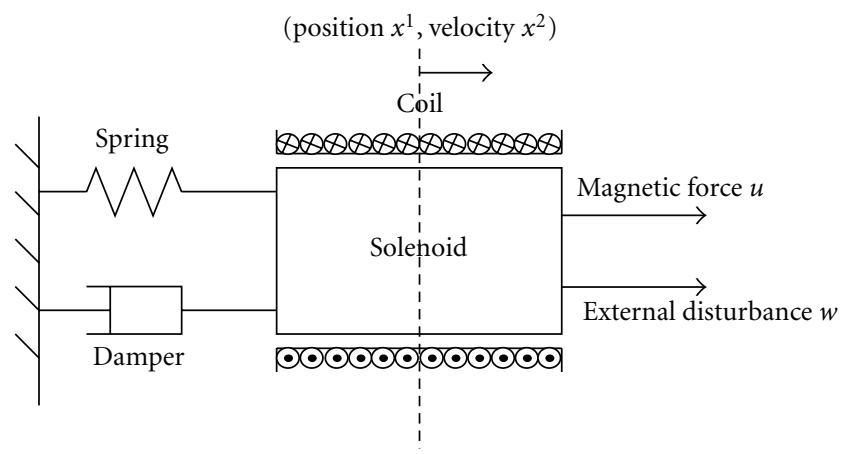

FIGURE 1: Solenoid system.

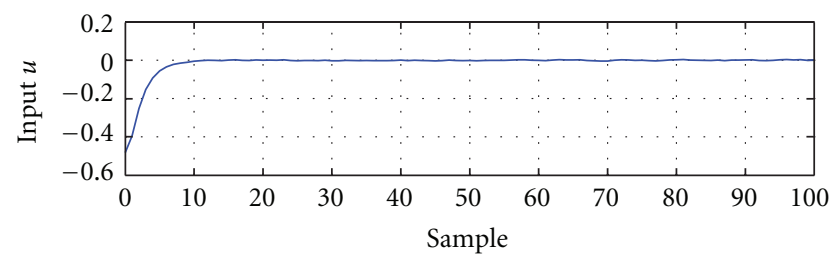

— Magnetic force $u$

(a)

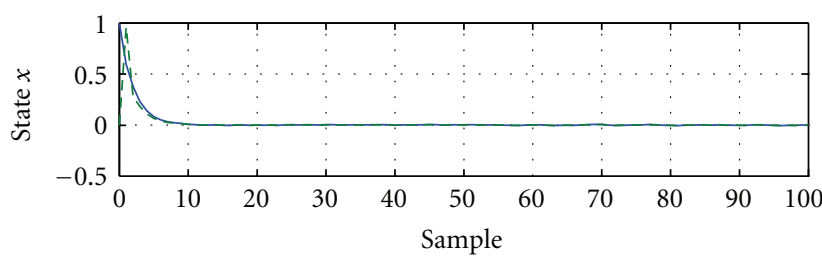

- Position $x^{1}$ --- Velocity $x^{2}$

(b)

Figure 2: Closed-loop response of the solenoid system with $\gamma^{2}=0.01$.

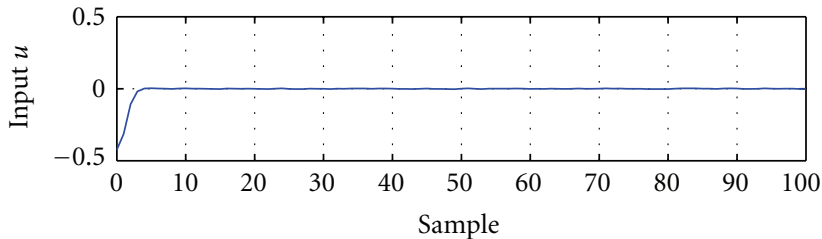

— Magnetic force $u$

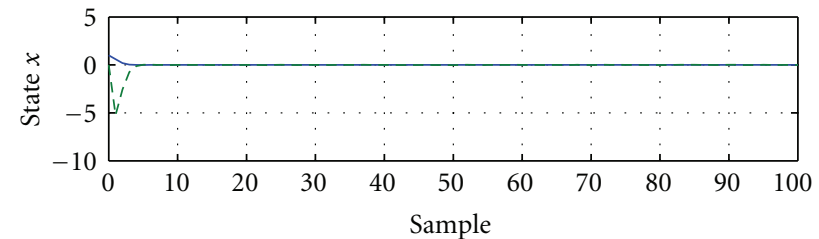

- Position $x^{1}$

-- Velocity $x^{2}$

(a)

(b)

FIgURE 3: Closed-loop response of the solenoid system with $\gamma^{2}=1$.

The model in terms of disturbance and perturbation vari- where ables is

$$
\begin{aligned}
x_{k+1}= & {\left[\begin{array}{cccc}
1 & 0 & 0.1 & 0 \\
0 & 1 & 0 & 0.1 \\
-0.1 \frac{K}{m_{1}} & 0.1 \frac{K}{m_{1}} & 1 & 0 \\
0.1 \frac{K}{m_{2}} & -0.1 \frac{K}{m_{2}} & 1 & 0
\end{array}\right] x_{k}+\left[\begin{array}{c}
0 \\
0 \\
\frac{0.1}{m_{1}} \\
0
\end{array}\right] u_{k} } \\
& +B_{w} w_{k}+B_{p} p_{k}, \\
q_{k}= & C_{q} x_{k}+D_{q u} u_{k}+D_{q w} w_{k}, \\
p_{k}= & \Delta q_{k}, \\
y_{k}= & {\left[\begin{array}{lll}
0 & 1 & 0
\end{array}\right] x_{k}, }
\end{aligned}
$$

$$
\begin{aligned}
& B_{w}=\left[\begin{array}{c}
0 \\
0.01 \\
0 \\
\\
0
\end{array}\right], \quad B_{p}=\left[\begin{array}{c}
0 \\
0 \\
-0.1 \\
0.1
\end{array}\right], \\
& C_{q}=\left[\begin{array}{lll}
0.475 & -0.475 & 0
\end{array}\right], \\
& D_{q w}=0, \quad D_{q u=0},
\end{aligned}
$$

where $x_{1}$ and $x_{2}$ are the positions of body 1 and 2, and $x_{3}$ and $x_{4}$ are their velocities, respectively. $m_{1}$ and $m_{2}$ 


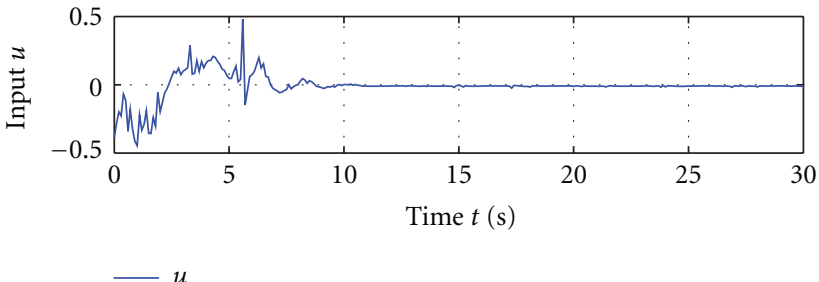

(a)

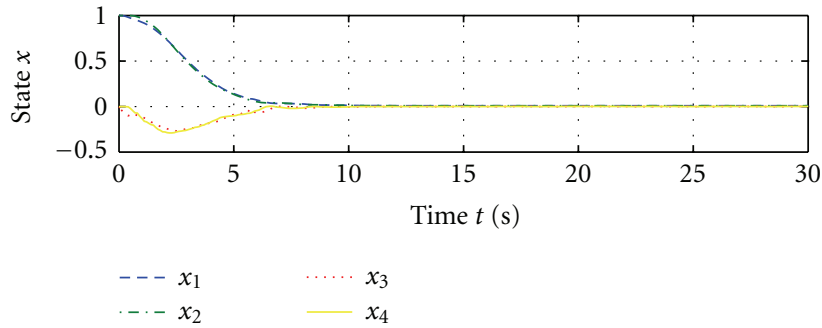

(b)

FIGURE 4: Closed-loop response of the coupled spring-mass system with $\gamma^{2}=0.45$.

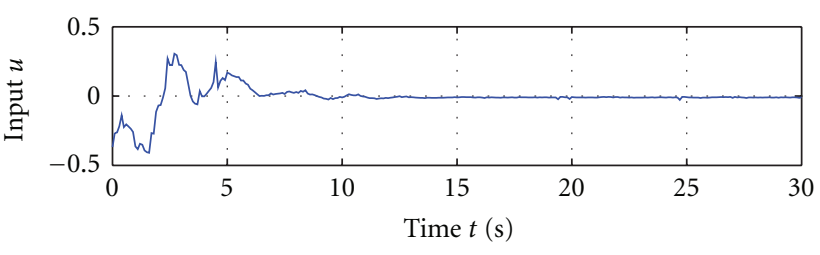

$-u$

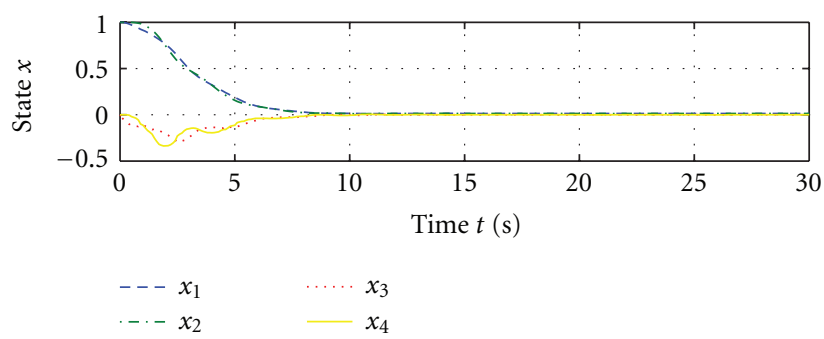

(b)

(a)

FIgURE 5: Closed-loop response of the coupled spring-mass system with $\gamma^{2}=4$.

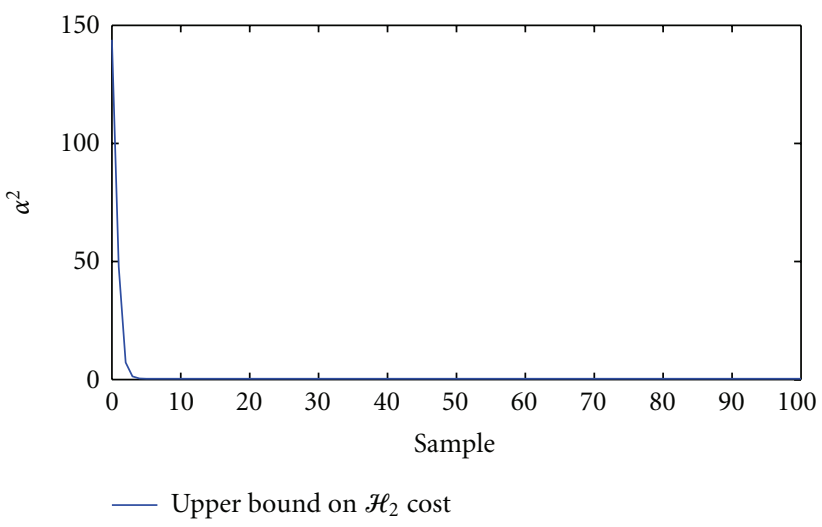

(a) System with $\gamma^{2}=0.01$

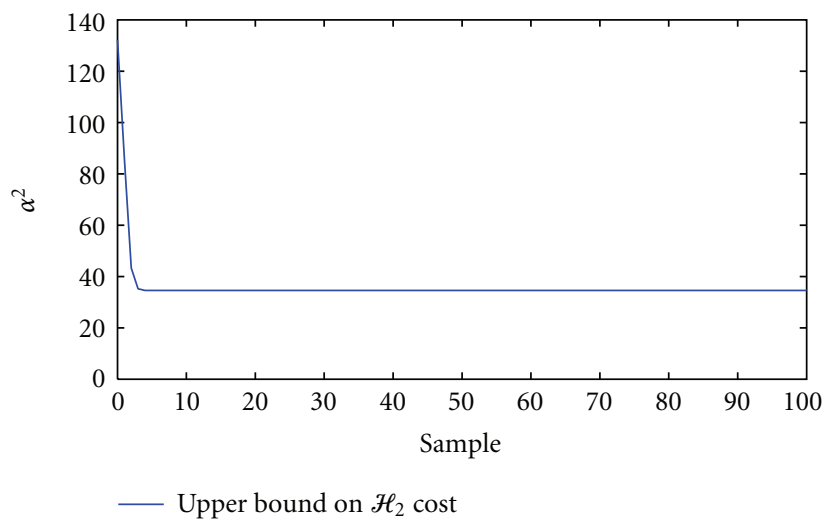

(b) System with $\gamma^{2}=1$

FIgURE 6: Upper bound on $\mathscr{H}_{2}$ cost function for solenoid system.

are the masses of the two bodies and $K$ is the spring constant. The initial state is given as $x_{0}=\left[\begin{array}{llll}1 & 1 & 0 & 0\end{array}\right]^{T}$. The cost function is specified using $C_{Z}=\operatorname{diag}(1,1,1,1)$, and $D_{z u}=1$. We consider the system with $m_{1}=m_{2}=1$ and $K \in[0.5,10]$.

A persistent disturbance of the form $w_{i}=0.1$ for all sample time was considered. Here we set $\gamma^{2}=0.45$ and $\gamma^{2}=$ 4. Figures 4 and 5 compare the closed-loop response for the high and low disturbance rejection levels, respectively, for randomly generated $\Delta$ 's. The value of $\gamma^{2}$ for high disturbance rejection was the lowest value for which a feasible solution exists. An input constraint of $\left|u_{k}\right| \leq 1$ is imposed, which is satisfied. The computation time for 300 samples was about $47 \mathrm{~s}$, making $0.16 \mathrm{~s}$ per sample.
4.3. Discussion. Note that the performance and response of the systems based on the high disturbance rejection level were better than those obtained using low disturbance rejection level, since the states and control are regulated to smaller steady state values. Constraints on the input were satisfied in both cases; however, the constraints were more conservative with respect to the control signal for the low disturbance rejection level. For example in the solenoid system, the control signal for high disturbance rejection level was 0.4826 and that for low disturbance rejection level was 0.4144. The issue of conservativeness in the mixed $\mathscr{H}_{2} / \mathscr{H}_{\infty}$ setting has been considered in [18]. For the systems considered, the upper bound on the $\mathscr{H}_{2}$ cost function $\alpha^{2}$ is depicted in Figures 6 and 7 for the high and low disturbance rejection 


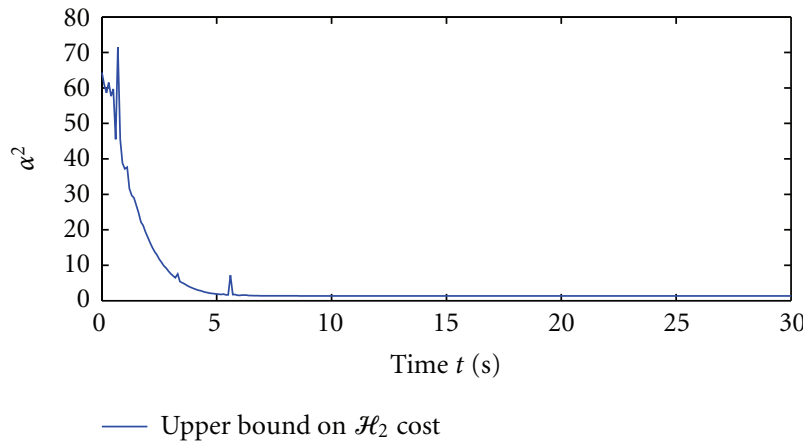

(a) System with $\gamma^{2}=0.45$

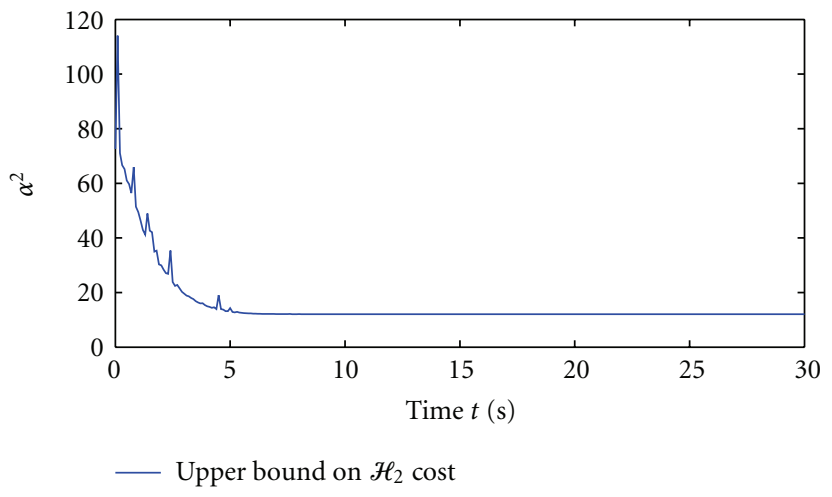

(b) System with $\gamma^{2}=4$

FIGURE 7: Upper bound on $\mathscr{H}_{2}$ cost function for coupled spring-mass system.

levels. It can been seen that the performance coefficient obtained for the high and low disturbance rejection levels is small. However, the offset level on the low disturbance level is higher than that for the high disturbance level. This is due to the higher value of $\gamma^{2}$ in $x_{0}^{T} P x_{0}+\gamma^{2} \bar{w}^{2}$. In Example 2, we have considered uncertainty in the model by using variable spring constant $K$.

\section{Conclusion}

In this paper, we proposed a robust model predictive control design technique using mixed $\mathscr{H}_{2} / \mathscr{H}_{\infty}$ for time invariant discrete-time linear systems subject to constraints on the inputs and states. This method takes account of disturbances naturally by imposing the $\mathscr{H}_{\infty}$-norm constraint in (14) and thus extends the work in [9] by the introduction of structured, norm-bounded uncertainty. The uncertain system was represented by LFTs. The development is based on full state feedback assumption and the on-line optimization involves the solution of an LMI-based linear objective minimization (convex optimization). Hence, the resulting state-feedback control law minimizes an upper bound on the robust objective function. The new approach reduces to that of [9] when there are no perturbations present in the system and to [7] when there are no disturbances. Thus, we have been able to show that it is possible to handle uncertainty and disturbance in the mixed $\mathscr{H}_{2} / \mathscr{H}_{\infty}$ model predictive control design approach. The two examples illustrate the application of the proposed method.

\section{Acknowledgments}

This was parts supported by Commonwealth Scholarship Commission in the United Kingdom (CSCUK) under Grant NGCS-2004-258. With regard to this paper, particular thanks go to Dr. Imad M. Jaimoukha and Dr. Eric Kerrigan.

\section{References}

[1] E. F. Camacho and C. Bordons, Model Predictive Control, Springer, 2nd edition, 2004.
[2] J. Richalet, A. Rault, J. L. Testud, and J. Papon, "Model predictive heuristic control. Applications to industrial processes," Automatica, vol. 14, no. 5, pp. 413-428, 1978.

[3] P. E. Orukpe, X. Zheng, I. M. Jaimoukha, A. C. Zolotas, and R. M. Goodall, "Model predictive control based on mixed $\mathscr{H}_{2} / \mathscr{H}_{\infty}$ control approach for active vibration control of railway vehicles," Vehicle System Dynamics, vol. 46, no. 1, pp. 151160, 2008.

[4] H. S. Witsenhausen, "A minimax control problem for sampled linear systems," IEEE Transactions on Automatic Control, vol. 13, no. 1, pp. 5-21, 1968.

[5] R. S. Smith, "Robust model predictive control of constrained linear systems," in Proceedings of the 2004 American Control Conference (AAC'04), pp. 245-250, July 2004.

[6] P. E. Orukpe and I. M. Jaimoukha, "A semidefinite relaxation for the quadratic minimax problem in $\mathscr{H}_{\infty}$ model predictive control," in Proceedings of IEEE Conference on Decision and Control, New Orleans, La, USA, 2007.

[7] M. V. Kothare, V. Balakrishnan, and M. Morari, "Robust constrained model predictive control using linear matrix inequalities," Automatica, vol. 32, no. 10, pp. 1361-1379, 1996.

[8] R. S. Smith, "Model predictive control of uncertain constrained linear systems: Lmi-based," Tech. Rep. CUED/F-INFENG/ TR.462, University of Cambridge, Cambridge, UK, 2006.

[9] P. E. Orukpe, I. M. Jaimoukha, and H. M. H. El-Zobaidi, "Model predictive control based on mixed $\mathscr{H}_{2} / \mathscr{H}_{\infty}$ control approach," in Proceedings of the American Control Conference (ACC '07), pp. 6147-6150, July 2007.

[10] P. E. Orukpe and I. M. Jaimoukha, "Robust model predictive control based on mixed $\mathscr{H}_{2} / \mathscr{H}_{\infty}$ control approach," in Proceedings of the European Control Conference, pp. 2223-2228, Budapest, Hungary, 2009.

[11] S. Boyd, L. El Ghaoui, E. Feron, and V. Balakrishnan, Linear Matrix Inequalities in Systems and Control Theory, SIAM Studies in Applied Mathematics, 1994.

[12] A. Packard and J. Doyle, "The complex structured singular value," Automatica, vol. 29, no. 1, pp. 71-109, 1993.

[13] K. Sun and A. Packard, "Robust $\mathscr{H}_{2}$ and $\mathscr{H}_{\infty}$ filters for uncertain LFT systems," in Proceedings of the 41st IEEE Conference on Decision and Control, pp. 2612-2618, Las Vegas, Nev, USA, December 2002.

[14] C. K. Li and R. Mathias, "Extremal characterizations of the Schur complement and resulting inequalities," SIAM Review, vol. 42, no. 2, pp. 233-246, 2000. 
[15] P. E. Orukpe, Model predictive control for linear time invariant systems using linear matrix inequality techniques, Ph.D. thesis, Imperial College, London, UK, 2009.

[16] H. Huang, L. Dewei, and X. Yugeng, "Mixed $\mathscr{H}_{2} / \mathscr{H}_{\infty}$ robust model predictive control based on mixed $\mathscr{H}_{2} / \mathscr{H}_{\infty}$ with normbounded uncertainty," in Proceedings of the 30th Chinese Control Conference, pp. 3327-3331, Yantai, China, 2011.

[17] D. Jia, B. H. Krogh, and O. Stursberg, "LMI approach to robust model predictive control," Journal of Optimization Theory and Applications, vol. 127, no. 2, pp. 347-365, 2005.

[18] P. E. Orukpe, "Towards a less conservative model predictive control based on mixed $\mathscr{H}_{2} / \mathscr{H}_{\infty}$ control approach," International Journal of Control, vol. 84, no. 5, pp. 998-1007, 2011. 

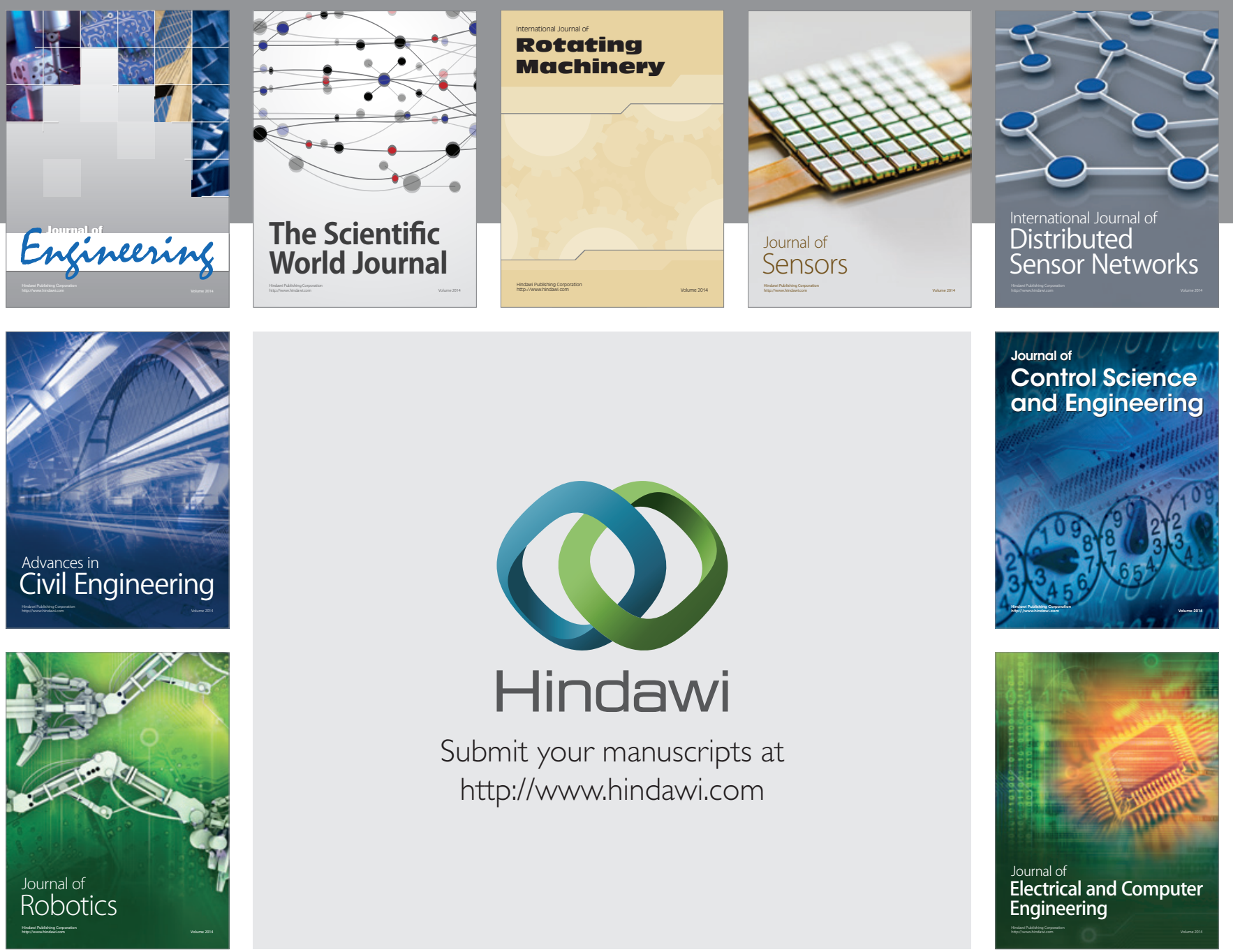

Submit your manuscripts at

http://www.hindawi.com
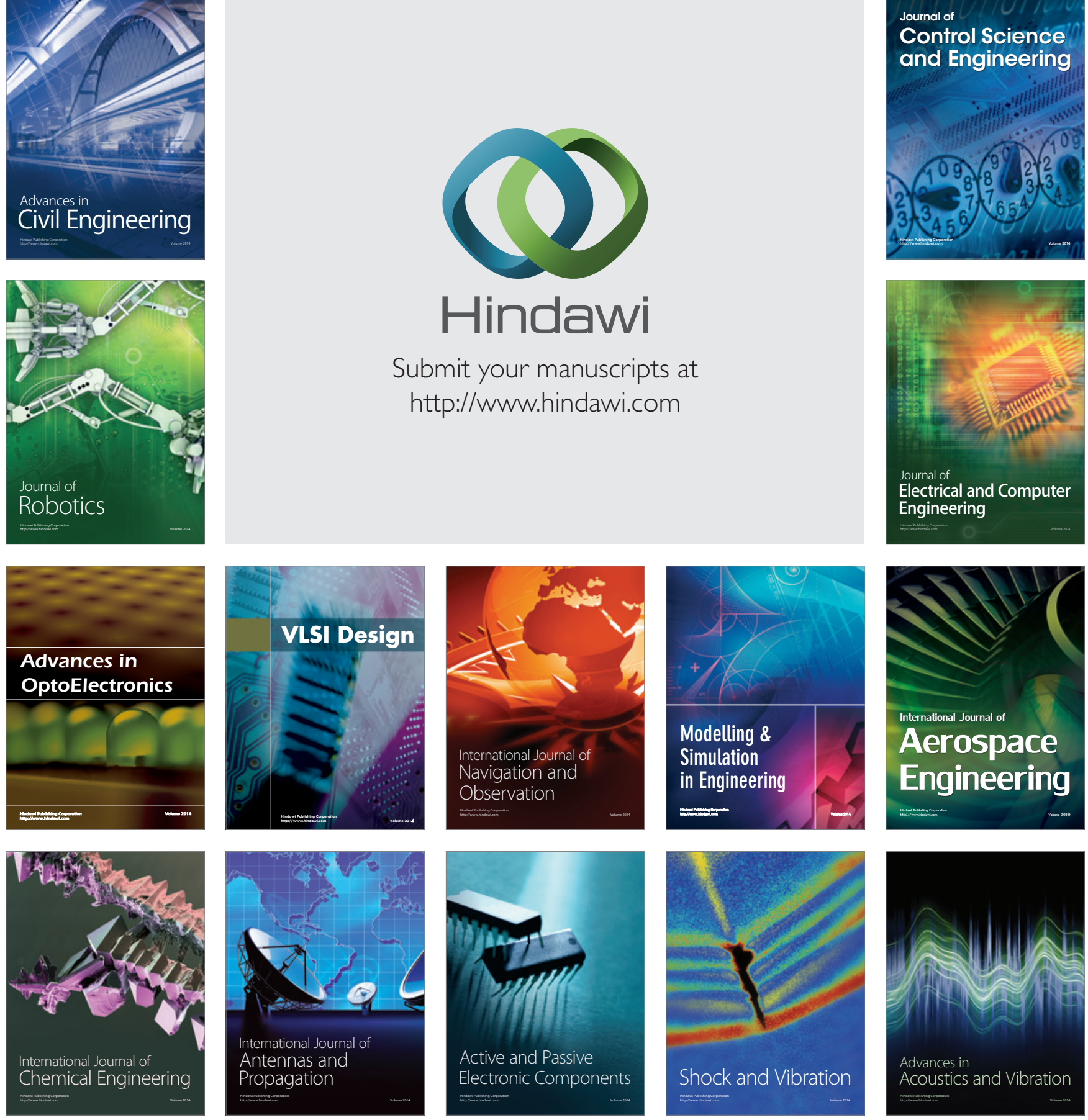